\title{
Clear aligners, the aesthetic solution: a review
}

\author{
Gopala Krishna Ganta1, ${ }^{*}$, Kamala Cheruvu², Rama Krishna Ravi ${ }^{3}$, Raghavendra Prasad Reddy ${ }^{4}$ \\ ${ }^{1}$ Masters' student in Health informatics, Indiana university Purdue university Indianapolis, Indiana, USA. \\ ${ }^{2}$ Post graduate student, Department of Orthodontics and Dentofacial Orthopedics, GITAM Dental College and \\ Hospital, Visakhapatnam, Andhra Pradesh, India, 530045. \\ ${ }^{3}$ Professor, Department of Conservative Dentistry and Endodontics, Mallareddy Dental College, Hyderabad, \\ Telangana, India. \\ ${ }^{4}$ Senior Manager, Medical Safety and Risk Management Servier Pharmaceuticals, Boston, MA 02210, USA.
}

\section{N F O R M A T I O N A B S T R A C T}

\section{Article History}

Received 28 ${ }^{\text {th }}$ February 2021

Accepted 12th June 2021

Available online

$1^{\text {st }}$ August 2021

\section{K E Y W O R D S}

\section{Aligners}

Polyurethane

PET

Thermoplastic materials

Stereolithography
In the recent past, the demand for adult orthodontic treatment has increased multiple folds. As a repercussion of this stipulation, invisible orthodontic treatment has expanded its horizon. Efforts of using aesthetic brackets, wires, and lingual techniques to meet the expectations of adult patients have been practiced. Though, these techniques seemed to be promising in the initial days, soon decreased in popularity due to drawbacks associated with them. Clear aligners were offered as a newer and superior aesthetic alternative to overcome the difficulties associated with prior aesthetic approaches. These aligners are made up of different thermoplastic materials intended to move the malpositioned teeth in the desired corrected position following push mechanics. This article deals with new generation orthodontic treatment using aligners. It deals with history, generations, different materials used, and the distances yet to be travelled to become the benchmark and replace the existing conventional braces. This paper also gives a bird's eye view of the method of aligner fabrication and the significant differences between the regular braces and aligners used in orthodontics.

\section{Introduction}

With the growing demand of an alluring and glamorous society, many teens, adolescents, and adults seeking orthodontic treatment are demanding appliances that are both more aesthetic and comfortable than conventional fixed appliances. The amassed demand for the more aesthetic orthodontic appliance has led to the revolution of invisible appliances such as ceramic brackets, lingual brackets, and aligners [1]. Among these appliances, aligners are desired over the other by adults as they exhibit superior aesthetics and give more comfort. Similar to fixed devices, aligners also embrace a wide range of applications in correcting various malocclusions. In the current market, there are different types of commercially available aligners. The common aspect among them is that they all are fabricated using clear thermoformed plastic, which covers many or all teeth. Thermoplastic materials are polymers that are either in the linear or slightly branched configuration. Strong covalent and weak Van der Waals bonds hold the different units of the plastic together. On heating, the molecular chains move and make the plastic flexible and pliable to any desired shape, and subsequently, they are cool down to room temperature. During cooling, the molecular chains solidify and retain their new shape [2]. 
Thermoplastic materials have been part of orthodontic treatments for decades, but their application was limited. However, with the advancement and progression of technology, these plastics can now be manipulated and used for correcting malocclusion [3]. These aligners are generally series of removable thermoplastic appliances that are custom-tailored, transparent, and esthetic alternatives to traditional metallic braces.

\section{History}

In the year 1945, Kesling fabricated retainers from wax setups of patients using rubber materials. Kesling witnessed that these rubber retainers did not just help in detailing the completed cases but also in minor tooth movement. He had predicted the possibility of considerable movements with these retainers. As Kesling's trial was successful, this led to the opening of a new window in the field of orthodontics [4].

Later in 1959, Dr Henry Nahoum attempted to refine the technique put forward by Kesling. He developed a method with a vacuum to fabricate the appliance, which displayed a firm and excellent adaptation to the model/cast. The process involved the fabrication of a plaster cast by correcting the rotations witnessed in the patient's anterior teeth. The rotated anterior teeth on the cast were sectioned using a goldsmith's saw or a fissure bur. The sectioned teeth were arranged with wax according to the final estimated and desired outcome. Then, the appliance was fabricated using rubber material that was vacuum formed over the designed model, and the excess material was removed $[5,6]$.

In 1971, Ponitz presented Invisible retainers using base plate wax on the prepositioned cast and suggested to cause limited tooth movements [7]. As time progressed, Sheridan and few others introduced interproximal tooth reduction and used a clear Essix appliance for facilitating proper tooth alignment [5]

Making impressions, pouring castings, and repeating the procedure a hundred times is not as simple as it appears. As a result, technology was used to make the method more convenient, such as the CAD-CAM (Computer-aided design computer-aided milling) technology. This technology uses graphics and predesigning the post-treatment customized models using software and executes it. This technology helped to eliminate the time-consuming process, to transform the slow weary process intolarge-scale production and a fast procedure [8]. As demand increased, investment in research and development (R\&D) further increased and became a critical step in developing an alternative to conventional braces.

\section{Types of materials}

Various thermoplastic materials that are currently used for fabrication include polyvinyl chloride, polyurethane (PU), polyethylene terephthalate (PET), and polyethylene terephthalate glycol (PETG) [9]

Polyurethane is a versatile material that allows a wide range of applications. PU is primarily composed of di- and tri-isocyanates, and polyols [10] also contain some additional materials to improve the properties of plastic. Because of its characteristics, the material is frequently used in aligners. PU has a higher resistance to compression and tension, is harder, and has a higher load-bearing capacity, making it a popular material. Under loads, PU material changes its shape but later attains its original shape when the load is removed. Because of the material's flexibility, it is able to elongate and recover. The material also exhibits high tear resistance and a wide range of resiliency. PU is an opaque substance in general, and its opacity increases during the mixing process, limiting its use in invisible orthodontics [11].

Polyethylene terephthalate (PET) is commonly known as polyester, which is made up of combining ethylene glycol with terephthalic acid. The material can be drawn into tapes, films or fibres [12]. The material is employed in various daily commodities as well as in the medical field. PET is frequently used as a substitute for grafts, vascular prostheses, etc. The PET exits in both amorphous and crystalline forms, which influence the properties. The amorphous structure is transparent, whereas the crystalline structure is opaque and white. Crystalline forms exhibit good strength, hardness and stiffness, and amorphous forms demonstrate superior ductility [13]. PET material can be either rigid or semi-rigid depending upon the processing methods employed. The material displays excellent mechanical properties, toughness, resistance against various solvents.

PETG is a non-crystalline co-polyester, which is made up of 1, 4 - cyclohexane two methanol (CHDM), ethylene glycol (EG) and terephthalic acid (TPA) [11]. The material shows excellent transparency, adequate flow property, and resistance against solvent. PETG is the 
material that can either be punched, die-cut, printed, hot stamped etc. PET-G is the choice of material to fabricate complex and intricate designs. The PET-G materials are exceptionally durable, have high impact strength, and are resistant to chemical changes. PETG is a transparent thermoplastic material and exhibits roughness that is lower than glass. Various bending tests suggested that the PETG is a very ductile material [11]. The PETG is a modified and altered form of PET that transforms its form from semicrystalline to amorphous form turning the material to be more transparent and esthetically appealing. The $\mathrm{Tg}$ of the material is around $80^{\circ} \mathrm{C}$, allowing better handling of the material with features that resemble glass [14]. This modified PET also exhibits altered optical and mechanical properties. The improved transparency, mechanical properties and optical properties make PETG a promising material in the fabrication of aligners.

To improve the properties of these materials and their influence in the fabrication of aligners and tooth movement, research is being conducted on these polymers in various manufacturing processes, blending of various thermoplastic materials, and in varied proportions of these materials.

\section{Types of aligners [15]}

The aligners are broadly classified as vacuum-formed, and pressure formed. Nahoum differentiated between vacuum formed and pressure-formed appliances. Both the appliances use air pressure for their fabrication, but they differ in their pressure levels. While vacuumformed uses a pressure around 3-14 psi, the pressure formed uses a pressure around 100 psi.

\subsection{Generations of aligners [15]}

The aligners have undergone many transformations to improve their efficiencies and treat various malocclusions more aesthetically, comfortably, and effectively.

\subsubsection{First-generation aligners}

These are supposed to be the initial or the earliest form of aligners. The results of the treatment were completely based upon the thermoformed plastics. No auxiliaries were attached to it.

\subsubsection{Second-generation aligners}

These aligners are manufactured along with the attachments to provide better tooth movement. The second-generation aligners also included the composite buttons on the teeth and inter-maxillary elastics.

The invention of newer generations have been undertaken to achieve better results and treat a larger spectrum of malocclusions.

\subsubsection{Third-generation aligners}

In the third-generation aligners, precision cuts, elastics, power ridges for lower anterior and upper incisors, optimal rotation attachment for premolars, and variation in canine attachments were available for treating class 2 and class 3 patients.

\subsubsection{Forth-generation aligners}

Next-generation smart-force, optimized root control attachments, a new multi-tooth approach for the open bite, new optimized multi-plane movements are included in the fourth generation.

\subsubsection{Fifth-generation aligners}

Fifth-generation aligners introduce the pressure area to allow for improved intrusion, enhanced deep bite attachments on premolars for extrusion, and bite ramps to generate posterior space.

\subsection{Fabrication of aligners}

For the fabrication of aligners, the pioneering methods are no longer used; instead, CAD-CAM is used. The dentist transmits the impression (either a 3D or a traditional impression) to the lab, which converts it into digital format (3D if a traditional impression is sent) and forecasts the treatment outcome back to the dentist. Once the dentist is satisfied with the preliminary result, the required amount of tooth movement to align them according to the agreed plan is calculated, and the thermoplastic trays are made accordingly. Every tray should exhibit around $1 / 10 \mathrm{~mm}$ of the desired movement. Once the number of trays required for the treatment has been determined, the trays are manufactured using a Rapid prototyping technology known as Stereo Lithography [16]. The method is exposing a laser to a photo-sensitive liquid resin, which cures the resin into a hard plastic-like consistency. Layer by layer, the operation is continued until the desired thickness is obtained.

Mild to moderate crowding, posterior dental expansion, tooth intrusion, molar distalization, aligning and levelling of arches, regulating anterior intrusion, controlling posterior buccolingual inclination, and Angle's class 1 , class 2 , and class 3 malocclusions are 
all indications for aligners.

Aligners may not be successful in cases with severely crowded teeth, growth discrepancies, impacted teeth, open bite, severely pointed teeth, extrusion of teeth, and teeth with short clinical crowns [17].

Compared to conventional braces, which are typically metal and exhibit metallic colour, aligners are clear/ invisible. Traditional braces must be worn 24 hours a day since they are fixed and cannot be adjusted by the patient, whereas aligners are detachable and worn for around 12-14 hours per day. Oral hygiene can be maintained with traditional braces by brushing them with bracket brushes and cleaning with water picks. However, with aligners, oral hygiene can be maintained by brushing and removing and cleaning the aligners with Lukewarm water. Conventional braces require a monthly check-up, whereas aligner trays are replaced every two weeks. Both traditional braces and aligners may need to be positioned or a retainer is worn primarily during nights post-treatment. The multiple benefits associated with traditional braces are more effective for complex cases, as aligners do not demand self-discipline. Limitations of traditional braces include persistent discomfort or soreness from wires and brackets until treatment is completed, tooth discolouration owing to poor dental care, difficulty in eating soft and sticky foods, and allergic responses in certain patients who have nickel allergies [18]. Compared to traditional fixed appliances, aligners are less uncomfortable, allow for better dental hygiene, and cause less root resorption [19].

The pull mechanism is used to move teeth in traditional orthodontics. In the case of aligners, the push mechanism is used instead of the conventional pull method. This push mechanism has also proven to be efficient. As a result, orthodontists must change their perspective from the pull mechanisms utilised in traditional braces to the push mechanisms employed in aligners.

The drawbacks of aligners include the fact that they are removable and hence do not apply continuous forces to the teeth. As a result, there may be a delay in tooth movement. Every meal necessitates the removal and reinsertion of the device. The success of the treatment largely depends upon the patient's compliance. Aligners cannot be used in treating skeletal class 2 and skeletal class 3 patients and may be limited to camouflage treatment as in traditional braces. The aligners in the oral cavity are constantly in contact with various beverages or colour staining liquids and solids, resulting in staining of the aligners and ultimately affect the aesthetics.

PU-based aligners are more susceptible to discolouration than PC- and PGET-based aligners [20]. Also, the physical properties of aligners alter if they are in the oral cavity for about 14 days. Microcracks, abraded surfaces, localized calcified biofilms, and loss of transparency are few to mention. Despite these minor changes in the properties, numerous studies observed no release of unreacted monomers from the aligner on storing it in artificial saliva for about 14 days. Therefore, it can be suggested that the aligners possess good chemical stability [21].

Intra-oral ageing also affects the mechanical properties of aligners include, imparts more brittleness, decreased wear resistance, attenuation of forces also reduced with an increase in aging of aligners. Many times, these aligners do not exhibit proper fit after some time in the oral cavity.

To improve retention, attachments are included. Increase the thickness of the material or add bevelled or ellipsoid attachments to the cast, ideally the bevel attachments, to improve retention. Customized attachments for each tooth are also effective, but they have yet to be adequately documented. The type of material utilised has an impact on retention [22].

In recent years, ramps have improved attachments, and power ridges have been utilised to repair significant malocclusions with better and more predictable results. If these few flaws are addressed, aligners have the potential to offer good outcomes and be an excellent alternative to traditional braces in orthodontics.

\section{Conclusion}

The use of trendy thermoplastic materials to treat malocclusion is becoming increasingly popular since they are user-friendly and offer promising outcomes. Aligners are among those rare procedures that minimise the psychological trauma that patients often experience during conventional treatment. Even among the esthetic-conscious population, aesthetic approval throughout treatment must lead to growing popularity. There are a few areas of concern where aligners have yet to be created to deliver superior results. If done correctly, these aligners would be a noble and successful breakthrough invention in the 
realm of orthodontics during this era.

Conflicts of interest: Authors declared no conflicts of interest.

Financial support: None

\section{References}

1. Rosvall M.D., Fields H.W., Ziuchkovski J., Rosenstiel S.F., and Johnston W.M. Attractiveness, acceptability, and value of orthodontic appliances. Am J Orthod Dentofacial Orthop. 2009;135(3): 276-e1. https://doi.org/10.1016/j.ajodo.2008.09.020

2. Hussein Shakir Saleh Al Noor, Sami Kadhum. AlJoubori. Comparison of the hardness and elastic modulus of different orthodontic aligners' materials. Int $\mathbf{J}$ Med Res Pharmaceutic Sci. 2018; 5(9), 19-25. http:// doi.org/10.5281/zenodo.1443358

3. Dasy H, Dasy A, Asatrian G, Rózsa NK, Lee HF, Kwak JH. Effects of variable attachment shapes and aligner material on aligner retention. Angle Orthod. 2015;85(6):934-940. https://doi.org/10.2319/091014$\underline{637.1}$

4. Phan X, Ling PH. Clinical limitations of Invisalign. J Can Dent Assoc. 2007;73(3):263-6.

5. Sheridan JJ, LeDoux W, McMinn R. Essix retainers: Fabrication and supervision for permanent retention. $\mathrm{J}$ Clin Orthod 1993;27:37-45.

6. Nahoum HI. The vacuum formed dental contour appliance. NY State Dent J. 1964; 9:385-90

7. Ponitz RJ. Invisible retainers. American Journal of Orthodontics;59(3):266-72. https://doi.org/10.1016/0002-9416(71)90099-6

8. Barone S, Paoli A, Razionale AV, Savignano R. Computational design and engineering of polymeric orthodontic aligners. Int J Numer Method Biomed Eng. 2017; 33(8):e2839.

https://doi.org/10.1002/cnm.2839

9. Lombardo L, Arreghini A, Maccarrone R, Bianchi A, Scalia S, Siciliani G. Optical properties of orthodontic aligners - spectrophotometry analysis of three types before and after aging. Prog Orthod. 2015 Dec;16 (1):1-8. https://doi.org/10.1186/s40510-015-0111-z

10. Iijima M, Kohda N, Kawaguchi K, et al., Effects of temperature changes and stress loading on the mechanical and shape memory properties of thermoplastic materials with different glass transition behaviours and crystal structures. Eur J Orthod, 2015; 37 (6):665-670. https://doi.org/10.1093/ejo/cjv013
11. Latko-Durałek P, Dydek K, Boczkowska A. Thermal, Rheological and Mechanical Properties of PETG/ rPETG Blends. J Polym Environ. 2019; 27, 26002606. https://doi.org/10.1007/s10924-019-01544-6

12. Laurence W. McKeen,6 - Polyester Plastics, Editor (s): Laurence W. McKeen,In Plastics Design Library, Permeability Properties of Plastics and Elastomers (Fourth Edition), William Andrew Publishing, USA, 2017, Pages 95-114.

https://doi.org/10.1016/B978-0-323-50859-9.00006-3

13. Lanaro M, Booth L, Powell SK, Woodruff MA. Electrofluidodynamic technologies for biomaterials and medical devices: melt electrospinning. In Electrofluidodynamic Technologies (EFDTs) for Biomaterials and Medical Devices 2018 Jan 1 (pp. 37-69). Woodhead Publishing. https://doi.org/10.1016/B978-0-08-101745-6.00003-7

14. Hassan MH, Omar AM, Daskalakis E, Hou Y, Huang B, Strashnov I, Grieve BD, Bártolo P. The Potential of Polyethylene Terephthalate Glycol as Biomaterial for Bone Tissue Engineering. Polymers. 2020;12 (12):3045. https://doi.org/10.3390/polym12123045

15. Hennessy J, Al-Awadhi EA. Clear aligners generations and orthodontic tooth movement. J Orthod. 2016; 43: 68-76.

https://doi.org/10.1179/1465313315Y.0000000004

16. Kulshrestha R. Clear Aligners vs Fixed Orthodontic Treatment - Where are we now? J Dent \& Oral Disord. 2017; 3(6): 1076. https://doi.org/10.26420/ jdentoraldisord.2017.1076

17. Joffe L. Invisalign: early experiences. J Orthod. 2003 Dec;30(4):348-52.

https://doi.org/10.1093/ortho/30.4.348

18. Miller KB, McGorray SP, Womack R, Quintero JC, Perelmuter M, Gibson J, et al. A comparison of treatment impacts between Invisalign aligner and fixed appliance therapy during the first week of treatment. Am J Orthod Dentofacial Orthop. 2007; 131: 302 e301-e309.

https://doi.org/10.1016/j.ajodo.2006.05.031

19. Fujiyama K, Honjo T, Suzuki M, Matsuoka S, Deguchi T. Analysis of pain level in cases treated with Invisalign aligner: comparison with fixed edgewise appliance therapy. Prog Orthod. 2014; 15: 64. https://doi.org/10.1186/s40510-014-0064-7

20. Liu CL1, Sun WT1, Liao W1, Lu WX1, Li QW1, Jeong Y1, Liu J1, Zhao ZH. Colour stabilities of three types of orthodontic clear aligners exposed to staining 
agents. Int J Oral Sci. 2016 Dec 16;8(4):246-253.

https://doi.org/10.1038/ijos.2016.25

21. Gracco A, et al. Short-term chemical and physical changes in invisalign appliances. Aust Orthod J. 2009 May;25(1):34-40.

22. Dalaie K, Ghaffari S. Importance of Attachments in Treatment with Clear Aligners: A Narrative Review. Journal of Dental School, Shahid Beheshti University of Medical Sciences. 2020;38(1):41-6. 